\title{
A FLEXIBLE CONTENT-BASED APPROACH TO ADAPTIVE IMAGE COMPRESSION
}

\author{
Alexander Wong and William Bishop \\ Department of Electrical and Computer Engineering \\ University of Waterloo
}

\begin{abstract}
Recent research in image compression has focused on lossy compression algorithms. However, the baseline implementations of such algorithms generally use a universal quantization process that results in poor image quality for certain types of images, particularly mixed-content images. This paper addresses this image quality issue by presenting a new algorithm that provides flexible and customizable image quality preservation by introducing an adaptive thresholding and quantization process based on content information such as edge and texture characteristics from the actual image. The algorithm is designed to improve visual quality based on the human vision system. Experimental results from the compression of various test images show noticeable improvements both quantitatively and qualitatively relative to baseline implementations as well as other adaptive techniques.
\end{abstract}

\section{INTRODUCTION}

The popularity explosion of digital photography and the Internet has especially brought to light the importance of image compression for efficient data storage and data transmission. Recent research in image compression has focused on lossy compression algorithms, which are capable of providing very high compression ratios by sacrificing visual information in an image. The most popular of these lossy compression algorithms are blocktransform based schemes that utilize cosine transforms and, more recently, wavelet transforms. A widely used standard for digital image compression in consumer devices such as personal computers and digital cameras is an algorithm standardized by the Joint Photographic Experts Group, commonly known as JPEG [1]. One of the major weaknesses of JPEG and other block-transform image coding schemes is that no specific adaptive compression scheme is defined in the standards. All images are treated the same regardless of content. However, no one scheme is ideal for all types of image content. Furthermore, the entire image content is processed using the same scheme regardless of the local content in a particular area. This is problematic as images often contain diverse image content, particularly mixed-content images containing a combination of photographs, text, and flat diagrams.

For quite some time, research has been conducted into adaptive image quality preservation techniques for lossy image compression schemes.
This research can be generalized into three main categories:

i) Quantization optimization [2-5]

ii) Frequency threshold optimization [6-7]

iii) Regions-of-Interest (ROI)-based optimization [8-10]

In general, most of these algorithms do not provide a fine level of granularity for image quality adjustment and customizability.

The main contribution of this paper is a new content- adaptive image quality preservation algorithm for lossy compression algorithms. The algorithm is versatile and can retain detail clarity for different types of images, including mixed-content images. Furthermore, it is designed to be highly customizable for use in specific applications. In this paper, the proposed improvement algorithm is described and explained in detail in Section 2. Experimental results are described comparing the proposed algorithm with the MPEG2 Test Model 5 adaptive quantization algorithm in Section 3. Finally, conclusions are drawn based on the results in Section 4.

\section{PROPOSED CONTENT-ADAPTIVE COMPRESSION ALGORITHM}

The image quality preservation algorithm proposed extends upon the concept of adaptive thresholding and quantization using content knowledge from the original uncompressed image. It is possible to extract important characteristics from the input image to aid with the process of discarding the least relevant information from the image as well as determining the level of quantization on different types of image content within a single image. Two of the most perceptually important characteristics of an image to the human vision system are edges and textures. Therefore, it is important to exploit these characteristics to preserve image quality while maintaining high compression performance. The proposed algorithm attempts to provide a robust algorithm that is versatile and customizable, making it highly suitable for maintaining overall image quality in images of various content types.

\subsection{Overview}

Given an initial uncompressed raster image $R$, this algorithm divides $R$ into $B$ partitions and finds an approximately optimal set of $n$ quantization values $Q$ and threshold matrix of $n$ threshold values $T$ for each partition, where $n$ is defined as the number of image representation components produced by the compression algorithm for each partition. For example, the JPEG standard produces 63 spatial frequency coefficients (since the DC quantization remains unchanged) that can be adaptively quantized 
and threshold for each $8 \times 8$ partition, and so $n=63$. A general overview of the algorithm is shown in Figure 1.

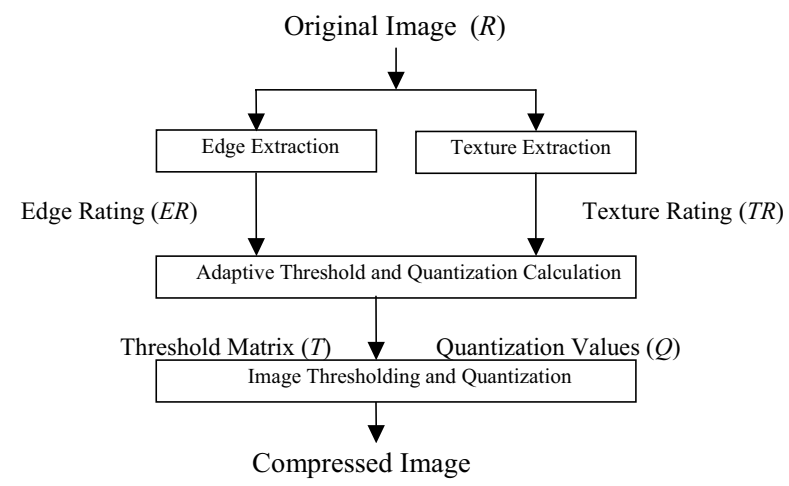

Figure 1. Content adaptive compression scheme

\subsection{Initial Setup}

For the proposed algorithm to function, a number of predefined matrices $T_{\min }, T_{\max }, Q_{\min }, Q_{\max }, I R_{\text {edge }}$ and $I R_{\text {texture }}$ must be constructed to use as reference models in the calculation of adaptive threshold matrix $T$ and quantization set $Q$ for each partition. $T_{\min }$ and $T_{\max }$ consist of the minimum and maximum allowable thresholds that can be applied to the image representation components respectively. $Q_{\min }$ and $Q_{\max }$ consist of the minimum and maximum allowable quantization step values that can be applied to the image representation components respectively. These threshold matrices and quantization sets are manually defined prior to compression based on the maximum and minimum compression rates desired for the input image. For example, the closer a threshold value in $T$ is to its corresponding value in $T_{\max }$, the more information is discarded and the higher the compression rate will be at the cost of image quality. Similarly, the closer a quantization value in $Q$ is to its corresponding value in $Q_{\max }$, the higher the compression rate will be at the cost of image quality. Finally, $I R_{\text {edge }}$ and $I R_{\text {texture }}$ represent the influence that edge characteristics and texture characteristics have over the calculation of the adaptive thresholds and quantization step values to be applied to the image respectively. For example, in realistic photographs, edge characteristics may have higher influence on quality preservation than texture characteristics. Values in $I R_{\text {edge }}$ and $I R_{\text {texture }}$ are in the range of $[-0.5,0.5]$, where a negative value indicates negative influence over the threshold and quantization value calculation for that component and a positive value indicates a positive influence. For example, negative values may be used for $I R_{\text {texture }}$ as complex textures can hide quantization errors better than smooth areas. Therefore, a negative value would mean that the higher the texture rating, the coarser the quantization steps. The setting of parameters for the $I R$ matrices is not discussed in detail in this paper, as it may vary depending on the domain in which the algorithm is used, as well as the file format used to store the image. For example, if the JPEG compression algorithm as specified in ISO/IEC IS 10918-1/3 [3] is used, then the influence matrices $I R_{\text {edge }}$ and $I R_{\text {texture }}$ must be constructed such that the influence a characteristic has on a frequency component must be the same for all frequencies. Furthermore, $Q_{\min }, Q_{\max }$ must be constructed such that $Q_{\min }$ is a positive scalar multiple of $Q_{\max }$.
The reason for this is that ISO/IEC IS 10918-3 specifies an extension to JPEG where a multiplier relative to the stored quantization matrix may be used to allow for adaptive coding.

\subsection{Edge and Texture Characteristics Extraction}

At this stage, the input image $R$ is processed using an edge detection algorithm to yield a binary edge map $E$, where 1 represents an edge pixel and 0 represents a non-edge pixel. An edge rating $E R$ in the range $[0,1]$ is calculated for each of the $B$ partitions:

$$
E R(b)=\left(E_{b} / N_{b}\right) / \max (E R)
$$

where $E_{b}$ is the number of edge pixels in partition $b$, and $N_{b}$ is the number of pixels in partition $b$. A high value of $E R$ indicates strong edge characteristics. A better representation of the image can be achieved utilizing the edge characteristics. For example, a partition with a high edge rating typically means that more data about the intermediate and high frequencies should be retained for better edge representation.

The gray-level co-occurrence hybrid structures [11] (GLCHS) $G_{\theta}$ for a pixel separation distance of $d=1$ and angles $\theta=0, \pi / 4, \pi / 2$, and $3 / 4 \pi$ are also derived for each of the $B$ partitions. GLCHS allows for faster calculation of co-occurrence statistics than the typical gray-level co-occurrence matrix (GLCM). An overall texture rating $T R$ in the range $[0,1]$ is calculated for each of the $B$ partitions based on the entropy of the calculated GLCHS:

$$
T R(b)=\max \left(E n t_{\theta=0}, E n t_{\theta=\pi / 4}, E n t_{\theta=\pi / 2}, E n t_{\theta=3 \pi / 4}\right) / \max (T R)
$$

where $E n t_{\theta}$ is the normalized entropy of the GLCHS $G_{\theta}$. A high value of $T R$ indicates complex texture characteristics. A better representation of the image can be achieved utilizing the texture characteristics since coarser quantization can be performed on regions with complex textures as such regions conceal quantization errors better than smooth regions, and vice versa.

\subsection{Adaptive Threshold and Quantization Set Computation}

After the edge and texture ratings have been determined, an adaptive threshold matrix $T$ and quantization set $Q$ are calculated for each of the partitions. For each partition in the image, the edge activity influence factor $E A I(f)$ is calculated for each of the $f$ image representation components:

$$
E A I(f)=E R(b) \times I R_{\text {edge }}(f)
$$

where $I R_{\text {edge }}(f)$ is the influence of edge characteristics on component $f$ and the $E R(b)$ is the edge rating for partition $b$. The texture complexity influence factor $T C I(f)$ is calculated for each of the $f$ image representation components:

$$
T C I(f)=T R(b) \times I R_{\text {texture }}(f)
$$

where $I R_{\text {texture }}(f)$ is the influence of texture characteristics on component $f$ and $T R(b)$ is the texture rating for partition $b$.

Finally, the adaptive threshold matrix $T$ and adaptive 
quantization set $Q$ are calculated as:

$$
\begin{gathered}
T(f)=T_{\max }(f)-\alpha(f)\left(T_{\max }(f)-T_{\min }(f)\right) \\
Q(f)=Q_{\max }(f)-\alpha(f)\left(Q_{\max }(f)-Q_{\min }(f)\right) \\
\alpha(f)=\max (\min (1 / 2+E A I(f)+T C I(f), 1), 0)
\end{gathered}
$$

where $E A I(f)$ is the edge activity factor at component $f$ for the selected partition, and $T C I(f)$ is the texture complexity influence factor at component $f$ for the selected partition. Limiting possible adaptive threshold and quantization values to minimum and maximum values helps ensure that potential errors that may occur during the edge and texture analysis stages do not lead to severe image degradation in the compressed image.

\subsection{Image Thresholding and Quantization}

Once the threshold matrix and quantization values for a particular partition have been computed, image thresholding and quantization is applied to the image representation components of the partition. For block-based image compression techniques such as the JPEG algorithm and tile-based wavelet algorithms such as Local Waveform Transform [12] and that proposed in JPEG2000 [13], the threshold matrices and quantization values are applied to the frequency or wavelet coefficients of each block or tile respectively. Finally, the information necessary to decompress the image is stored along with the image file as specified by the corresponding file format.

\section{EXPERIMENTAL RESULTS}

A comparison between the proposed algorithm and the adaptive quantization approach proposed in MPEG2 Test Model 5 as applied to a single image [14] was conducted quantitatively and qualitatively using the JPEG compression algorithm with $8 \times 8$ blocks. The standard Q-factor scaling approach used to control compression ratios in the JPEG standard was included as the basis for comparisons. The proposed algorithm was implemented using the Canny edge detection algorithm with the maximum and minimum threshold matrices and quantization values configured such that the compression rate of a particular test image was within $5 \%$ of that produced by the Q-factor scaling approach at different compression rates. The algorithms were tested on a variety of different images, from visually simple images such as Lena to visually complex images such as Mandrill. All images were represented in 8-bit grayscale and ranged from $256 \times 256$ to
$1024 \times 1024$ in size. For a quantitative comparison, the PSNR of the compressed images was measured at different compression rates.

The results for Lena are shown in Figure 2. It is observed from the quantitative measurements that the images produced using the proposed algorithm have approximately equivalent PSNR values when compared to the standard Q-factor scaling approach. On the other hand, the PSNR of the MPEG2 algorithm was consistently lower than the Q-factor algorithm and the proposed algorithm. Although PSNR is not a good measure in overall image quality, it is a reasonable quantitative measure for the image content preservation abilities of an algorithm.

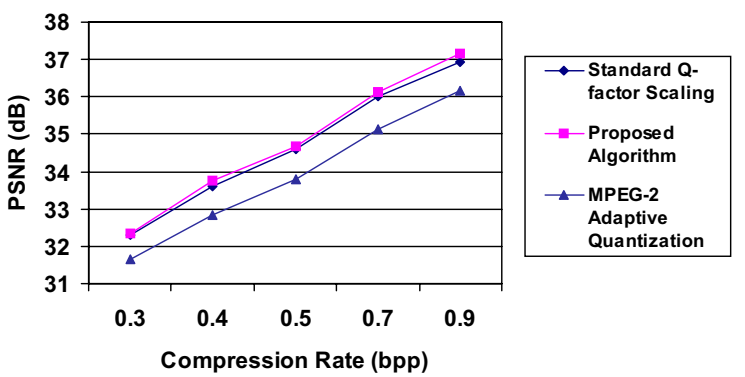

Figure 2. Comparison of different algorithms for Lena

The subjective results for specific regions in Lena are shown in Figure 3. From a subjective comparison of the resultant images, it can be observed that the overall quality of the images produced using the proposed algorithm is noticeably improved over the standard Q-factor approach. The edges are noticeably sharper and cleaner in the images produced using the proposed algorithm, as evident by the clarity in Lena's eye, eyebrow, and in the brim of her hat in Figure 3. It can also be observed that the image produced using the proposed algorithm has noticeably fewer blocking artifacts than the standard Q-factor approach. While the MPEG2 algorithm produced images that had fewer blocking artifacts than the proposed algorithm, the details of the image are noticeably inferior to that produced by the proposed algorithm. Detail clarity is of great importance to image quality preservation, as image quality degradation that is not related to image clarity such as blocking artifacts can be effectively removed during postprocessing, whereas degradation related to image clarity cannot be easily remedied due to the loss of information about these details. Therefore, the proposed algorithm strikes a balance between detail clarity and reduced blocking artifacts. Better image quality and compression performance can be achieved with further refinement of the predefined matrices used to determine the adaptive threshold and quantization values, particularly when applied to specific domains. 


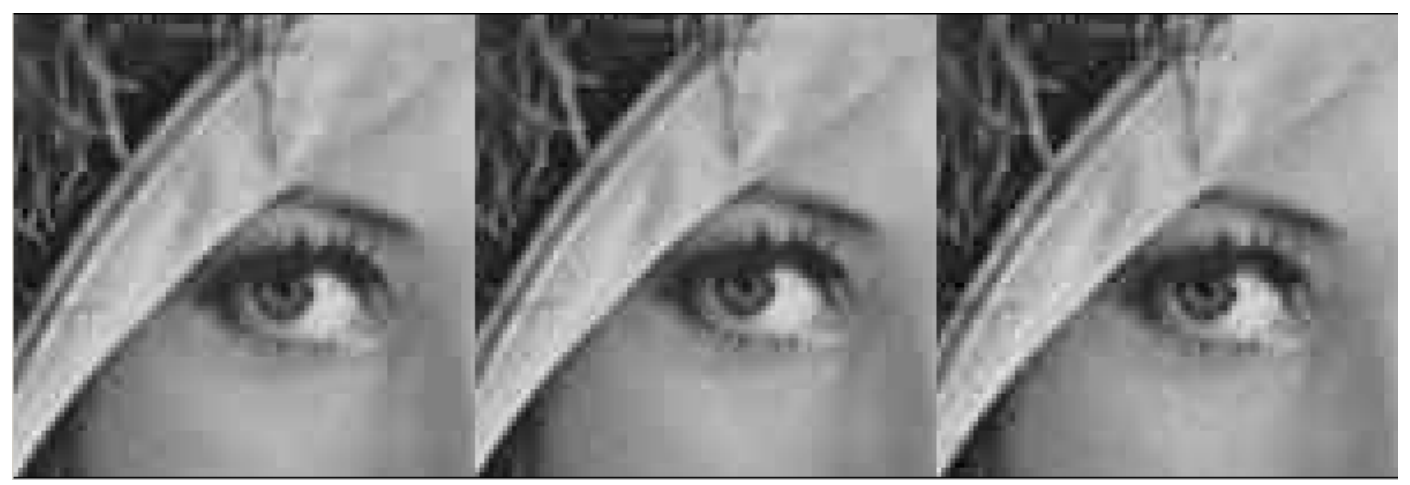

Figure 3. Comparison of Lena images generated using three different approaches

Left: Q-factor approach at $0.4 \mathrm{bpp}$

Center: Proposed approach at $0.4 \mathrm{bpp}$

Right: MPEG2 algorithm at 0.4 bpp

\section{CONCLUSIONS}

This paper has proposed a new method for image quality preservation based on the concept of adaptive thresholding and quantization using image content characteristics. The proposed algorithm is versatile and highly customizable for specific domains. Experimental results show that overall image quality preservation is noticeably improved over the Q-factor approach and the MPEG2 adaptive quantization algorithm at the same compression levels. It is believed that this method can be successfully implemented in various digital imaging systems such as digital cameras and multimedia systems to produce results with better overall visual quality preservation when lossy image compression is utilized. Future work includes the design and implementation of the proposed algorithm in hardware, as well as an investigation of optimal parameters for the algorithm in different domains.

\section{ACKNOWLEDGEMENTS}

This research has been sponsored in part by Epson Canada and the Natural Sciences and Engineering Research Council of Canada.

\section{REFERENCES}

[1] G. Wallace, "The JPEG Still Picture Compression Standard," Comm. ACM, Vol. 34, No. 4, pp. 30-34, 1991.

[2] D. Monro and B. Sherlock, "Optimum DCT Quantization," in Proc. DCC, pp. 188-194, March 30 - April 21993.

[3] R. Rosenholtz and A. Watson, "Perceptual adaptive JPEG coding," in Proc. ICIP, Vol. 1, pp. 901-904, Sept 1996.

[4] M. Ramos and S. Hemami, "Edge-adaptive JPEG Image Compression," in Proc. VCIP, Orlando, FL, pp. 465-474, 1996.

[5] M. Grazia Albanesi, M. Ferretti, F. Guerrini, "Adaptive image compression based on regions of interest and a modified contrast sensitivity function," in Proc. ICPR, Vol. 3, pp. 215-218, 2000.

[6] M. Ramos and R. Queiroz, "Adaptive Rate-Distortion-Based Thresholding: Application in JPEG Compression of Mixed Images for Printing," in Proc. ICASSP, Vol. 5, pp. 2431-2434, 1999.
[7] A. Zaid, O. Alata, F. Marmoiton, and C. Olivier, "Transform Image Coding with Global Thresholding: Application to Baseline JPEG”, in Proc. SIBGRAPI, pp. 164-171, October 2001.

[8] S. Gokturk, C. Tomasi, B. Girod, and C. Beaulieu, "Medical Image Compression Based on Region of Interest, with Application to Colon CT Images," in Proc. EMBC, Istanbul, Turkey, Vol. 3, pp. 2453-2456, October 2001.

[9] D. Nistér and C. Christopoulos, "Lossless Region of Interest with a Naturally Progressive Still Image Coding Algorithm," in Proc. ICIP, Vol. 3, pp. 856-860, 1998.

[10] M. Petrou, H. Peixin, S. Kamata, and C. Underwood, "Region-based image coding with multiple algorithms," in IEEE Trans. Geoscience and Remote Sensing, Vol. 39, No. 3, pp. 562570, 2001.

[11] D. Clausi and Y. Zhao, "Rapid Co-occurrence Texture Feature Extraction using a Hybrid Data Structure," in Computers \& Geosciences, Vol. 28, No. 6, pp. 763-774, 2002.

[12] B. Masschelein, "Local Wavelet Transform: a Cost-efficient Custom Processor for Space Image Compression," in Proc. SPIE Applications of Digital Image Processing, pp. 334-345, 2002.

[13] M. Boliek, C. Christopoulos, and E. Majani, "JPEG 2000 Part I Final Committee Draft Version 1.0," 2000, available online at http://www.jpeg.org/public/fcd15444-1.pdf.

[14] ISO/IEC JTC1/SC29/WG11/N0400, “Test Model 5”, 1993. 